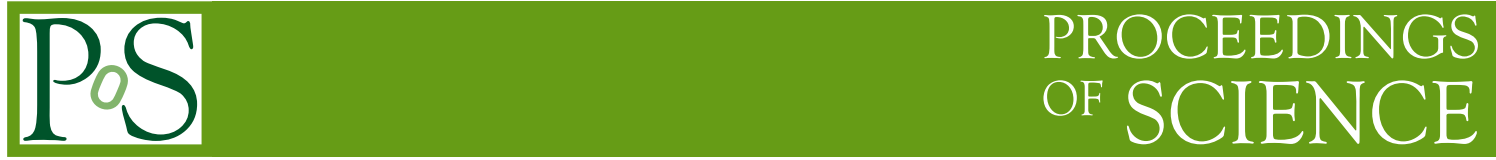

\title{
Transfer Matrix for Partially Quenched QCD
}

\section{Claude Bernard}

Department of Physics, Washington University, St. Louis, MO 63130, USA

E-mail: cb@lump.wust 1.edu

\section{Maarten Golterman*}

Department of Physics and Astronomy, San Francisco State University, San Francisco, CA

94132, USA

E-mail: maartenestars.sfsu.edu

We construct the transfer matrix for the ghost sector of partially quenched QCD. This transfer matrix is not hermitian, but we show that it is still bounded. We thus expect that all euclidean correlation functions will decay exponentially with distance (up to possible powers), and demonstrate that this is indeed the case for free ghost quarks.

The XXVIII International Symposium on Lattice Field Theory, Lattice2010

June 14-19, 2010

Villasimius, Italy

\footnotetext{
*Speaker.
} 


\section{Introduction}

The use of "partially quenched" QCD (PQQCD), in which valence and sea quark masses are varied independently, has become common practice in large-scale computations of hadronic quantities from QCD using lattice methods. This usefulness arises from the following observations:

1. Sea quark masses are, by definition, the quark masses that go into the generation of dynamical gauge field configurations on which hadronic quantities are evaluated. Valence quark masses are, by definition, the quark masses that go into the operators that are evaluated on these gauge configurations. Such operators are contractions of quark propagators, which depend on the gauge field configurations. For many applications, it is numerically less expensive to generate quark propagators than gauge field configurations. Given limited resources it can thus be advantageous to generate data for many values of the valence quark masses, using configurations depending on only a limited number of sea quark mass values.

2. PQQCD with a given number of sea quarks contains full QCD with the same set of sea quarks [1]. It follows that the low-energy constants (LECs) of the low-energy effective theory are those of the real world, because by construction, these LECs are independent of the quark masses [2]. ${ }^{1}$ For the case of unquenched QCD, this low-energy theory is chiral perturbation theory (ChPT), which provides a systematic expansion in (light) quark masses, with coefficients characterized by these LECs.

3. On the lattice, PQQCD can be generalized to "mixed-action" QCD, in which not only valence and sea quark masses are independently chosen, but also the discretization of the Dirac operator is chosen independently in the sea and valence sectors of the theory. This is a generalization in the sense that the continuum limit of such a lattice theory is described by PQQCD [3].

In order to apply these ideas, one needs the correct effective theory for PQQCD, i.e., one needs the partially quenched version of ChPT (PQChPT) [4]. While it is relatively straightforward to extend ChPT to the partially quenched case, it is less clear than in the unquenched case whether indeed PQChPT is the correct effective theory for PQQCD. This is because PQQCD violates a number of basic properties of a healthy quantum field theory on which arguments that ChPT is the correct effective theory for unquenched QCD rely. This follows from the definition of PQQCD as a euclidean path integral that includes an integral over ghost quarks, which have the same quantum numbers as the valence quarks, but the opposite (and thus "wrong") statistics.

For many field theories, the path integral for a euclidean theory can be expressed in terms of a transfer matrix. If the field theory is healthy, this transfer matrix is hermitian and bounded, and contact with the hamiltonian formulation of the theory can be made. ${ }^{2}$ But if the theory contains fields with the wrong statistics, it is not clear what properties of such a construction survive.

In Ref. [5], the validity of ChPT as a systematic low-energy effective theory for the Goldstone sector of QCD was conjectured to follow from the basic properties of a healthy quantum field

\footnotetext{
${ }^{1}$ They do depend on the number of sea quark flavors.

${ }^{2}$ Of course. quantum field theories exist which are believed to be healthy, but for which no transfer matrix can easily be constructed.
} 
theory: analyticity, unitarity, cluster decomposition, and symmetry. Stated differently, the $S$-matrix calculated with the most general local lagrangian consistent with a certain symmetry group was conjectured to be the most general possible $S$-matrix consistent with these basic properties. This was then used as a starting point for the systematic development of ChPT as an expansion of $S$ matrix elements in terms of the pion momenta, following a well-defined power-counting scheme. The reliance of this argument on unitarity, though, appears to be a fundamental difficulty in trying to extend it to the partially quenched case, which is certainly not unitary.

An alternative justification for ChPT as the low-energy effective theory for QCD was presented in Ref. [6]. The argument detailed there was based on locality and clustering of the underlying theory of unquenched QCD, as well as its symmetries. Locality and clustering guarantee the existence of vertices in the effective theory that are independent of the correlation functions in which they appear, and, consequently, the existence of a loop expansion. This approach seems more fruitful for PQQCD than that of Ref. [5]. By construction, PQQCD is local. It is less clear that it also satisfies the cluster property, but numerical evidence suggests that that is indeed the case, because euclidean correlation functions are observed to decay exponentially in distance (up to possible nonstandard powers, which come from the characteristic double poles [1,7] in the theory).

The first requirement for the chiral theory of PQQCD is, of course, the dynamical breaking of chiral symmetry in the theory with massless quarks. The Goldstone bosons associated with this breaking provide the low-energy degrees of freedom for the low-energy effective theory. It was argued in Ref. [7] that chiral symmetry must also be broken in PQQCD. The argument is that this must happen in the sea sector of that theory, because it is identical to unquenched QCD. This follows, since, by construction, the valence (and ghost) quarks are not part of the dynamics. Goldstone bosons corresponding to pions made only out of sea quarks thus have to be present in the partially quenched theory. Furthermore, the vectorlike partially quenched symmetries that relate sea and valence quarks then imply that there also have to be Goldstone excitations in the valence and ghost sectors, thus providing the necessary degrees of freedom for the construction of PQChPT. $^{3}$

The key outstanding issue for the justification of $\mathrm{PQChPT}$ as the correct chiral theory for PQQCD therefore seems to be the question of whether PQQCD obeys the clustering property. Here we begin an investigation into the clustering property of PQQCD by considering the construction of a transfer matrix for PQQCD. We start with the ghost sector, which is the ultimate source for the nonstandard features of the partially quenched theory.

\section{Staggered ghosts}

PQQCD contains three types of quarks: sea quarks, valence quarks, and ghost quarks, which have the same masses as the valence quarks, but opposite statistics. Because of this quark content, the complete fermion determinant of the partially quenched theory is just that coming from the sea sector, while the valence and ghost determinants cancel each other.

Since ghost quarks violate spin-statistics, we expect that obstructions to the existence of a well-behaved transfer matrix would originate from this sector of the theory. We therefore consider

\footnotetext{
${ }^{3}$ These vectorlike symmetries are not spontaneously broken [8].
} 
a theory of bosonic staggered quarks, in an arbitrary gauge field background. ${ }^{4}$ If we choose

$$
\begin{aligned}
S & =\sum_{x, y} \chi^{\dagger}(x) D(x, y) \chi(y)+\sum_{x} m \chi^{\dagger}(x) \chi(x) \\
& =\sum_{x}\left\{\frac{1}{2} \sum_{\mu} \eta_{\mu}(x)\left(\chi^{\dagger}(x) U_{\mu}(x) \chi(x+\mu)-\chi^{\dagger}(x+\mu) U_{\mu}^{\dagger}(x) \chi(x)\right)+m \chi^{\dagger}(x) \chi(x)\right\},
\end{aligned}
$$

with

$$
\eta_{\mu}(x)=(-1)^{x_{1}+\ldots+x_{\mu-1}}
$$

then

$$
Z=\int D \chi^{\dagger} D \chi \exp (-S)
$$

is convergent for $m>0$, because the staggered Dirac operator $D$ is anti-hermitian. The field $\chi$ is bosonic, i.e., it is a $c$-number valued staggered quark field. Of course, $S$ exhibits the same species doubling as the normal staggered quark action, and therefore we employ a two time-slice method for constructing the transfer matrix representation of $Z$, guided by the construction in Ref. [10].

We split $\chi$ into its real and imaginary parts by defining $\chi(x)=\eta_{4}(x) \phi_{1}(x)+i \phi_{2}(x)$, and then identify

$$
\begin{array}{cll}
t=2 k: & \phi_{1}(\vec{x}, t)=\Phi_{1, k}(\vec{x}), & \phi_{2}(\vec{x}, t)=-\Phi_{2, k}(\vec{x}), \\
t=2 k+1: & \phi_{1}(\vec{x}, t)=\Pi_{2, k}(\vec{x}), & \phi_{2}(\vec{x}, t)=\Pi_{1, k}(\vec{x}) .
\end{array}
$$

Then, if the extension of the (periodic) lattice in the time direction is $T$ (which we take to be even), the partition function can be written as the trace of the $T / 2$-th power of a transfer matrix,

$$
Z(\mathscr{U})=\operatorname{Tr}\left(\prod_{k=1}^{T / 2} \hat{T}_{k}(\mathscr{U})\right)
$$

in which

$$
\hat{T}_{k}(\mathscr{U})=e^{-\hat{\Phi}_{1} \mathscr{H}_{-}\left[\mathscr{U}(2(k+1)] \hat{\Phi}_{2}\right.} e^{-\hat{\Pi}_{2} \mathscr{H}_{+}[\mathscr{U}(2 k+1)] \hat{\Pi}_{1}} .
$$

Here $\mathscr{H}_{ \pm}$are defined through

$$
\begin{aligned}
& \Psi_{1} \mathscr{H}_{ \pm}[\mathscr{U}(t)] \Psi_{2}=\sum_{\vec{x}}\{ \pm \sum_{j} i \eta_{j}^{\prime}(\vec{x})\left(\Psi_{1}(\vec{x}) \operatorname{Re} U_{j}(\vec{x}, t) \Psi_{2}(\vec{x}+\vec{j})+(1 \leftrightarrow 2)\right) \\
&-\sum_{j} i \eta_{j}(\vec{x})\left(\Psi_{1}(\vec{x}) \operatorname{Im} U_{j}(\vec{x}, t) \Psi_{1}(\vec{x}+\vec{j})-(1 \rightarrow 2)\right) \\
&\left.+m\left(\Psi_{1}(\vec{x})^{2}+\Psi_{2}(\vec{x})^{2}\right)\right\} \\
& \eta_{j}^{\prime}(\vec{x})=\eta_{j}(\vec{x}) \eta_{4}(\vec{x})
\end{aligned}
$$

and the hermitian operators $\hat{\Phi}_{1,2}$ and $\hat{\Pi}_{1,2}$ satisfy the commutation rules

$$
\left[\hat{\Phi}_{a}(\vec{x}), \hat{\Pi}_{b}(\vec{y})\right]=i \delta(\vec{x}-\vec{y}) \delta_{a b} .
$$

\footnotetext{
${ }^{4}$ We use staggered quarks here because the nonperturbative definition of PQQCD with staggered quarks is straightforward. For other types of quarks, see Ref. [4] and refs. therein, in particular Ref. [9].
} 
From $\hat{T}$, we can define a hamiltonian in the limit in which the temporal lattice spacing goes to zero; with

$$
\begin{aligned}
& \hat{H}[\mathscr{U}(t)]=\lim _{a_{t} \rightarrow 0}-\log \hat{T}_{k}(\mathscr{U}) /\left(2 a_{t}\right), \\
& \hat{H}[\mathscr{U}(t)]= \hat{H}_{1}+i \hat{H}_{2}[\mathscr{U}(t)], \\
& \hat{H}_{1}=\frac{1}{2} m \sum_{\vec{x}}\left(\hat{\Pi}_{1}^{2}(\vec{x})+\hat{\Pi}_{2}^{2}(\vec{x})+\hat{\Phi}_{1}^{2}(\vec{x})+\hat{\Phi}_{2}^{2}(\vec{x})\right), \\
& \hat{H}_{2}[\mathscr{U}(t)]=\frac{1}{2} \sum_{\vec{x}, j}\left\{\eta_{j}^{\prime}(\vec{x})\left(\hat{\Pi}_{2}(\vec{x}) \operatorname{Re} U_{j}(\vec{x}, t) \hat{\Pi}_{1}(\vec{x}+\vec{j})-\hat{\Phi}_{1}(\vec{x}) \operatorname{Re} U_{j}(\vec{x}, t) \hat{\Phi}_{2}(\vec{x}+\vec{j})\right)\right. \\
& \quad-\eta_{j}(\vec{x})\left(-\hat{\Pi}_{1}(\vec{x}) \operatorname{Im} U_{j}(\vec{x}, t) \hat{\Pi}_{1}(\vec{x}+\vec{j})+\hat{\Phi}_{1}(\vec{x}) \operatorname{Im} U_{j}(\vec{x}, t) \hat{\Phi}_{1}(\vec{x}+\vec{j})\right) \\
&\quad+(1 \leftrightarrow 2)\} .
\end{aligned}
$$

Both $\hat{H}_{1}$ and $\hat{H}_{2}$ are hermitian, and do not commute. Therefore, $\hat{H}$ is not hermitian, and not normal. It follows that the transfer matrix is not hermitian and not positive definite.

Despite these (nonsurprising) conclusions, it is possible to make progress. Our transfer matrix factorizes as

$$
\hat{T}_{k}(\mathscr{U})=\hat{T}_{1}(\mathscr{U}) \hat{T}_{2}(\mathscr{U}),
$$

in which $\hat{T}_{1}$ is the exponential operator with $\mathscr{H}_{-}$and $\hat{T}_{2}$ is that with $\mathscr{H}_{+}$. Both $\hat{T}_{1}$ and $\hat{T}_{2}$ are normal and bounded. Since the hamiltonians $\mathscr{H}_{-}$and $\mathscr{H}_{+}$have a positive real part (the parts proportional to $m$ ):

$$
\left\|\hat{T}_{1,2}\right\| \leq 1
$$

which implies

$$
\|\hat{T}\| \leq\left\|\hat{T}_{1}\right\|\left\|\hat{T}_{2}\right\| \leq 1
$$

which establishes that all eigenvalues of $\hat{T}$ have an absolute value smaller than one. ${ }^{5}$ It follows that correlation functions in this theory decay exponentially with distance if the eigenvalue $\lambda_{0}$ with maximal $\left|\lambda_{0}\right|$ is unique. Of course, the construction needs to be extended to the complete partially quenched theory, but we do not expect any difficulties with the other building blocks of a complete transfer matrix.

\section{Trivial gauge field background}

For a trivial gauge field background, it is straightforward to diagonalize $\hat{H}$. In terms of canonical creation and annihilation operators, introduced through

$$
\begin{aligned}
& \Phi_{1}(\vec{x})=\int \frac{d^{3} k}{(2 \pi)^{3}} \frac{1}{\sqrt{2}}\left(a_{1}(\vec{k})+a_{1}^{\dagger}(-\vec{k})\right) e^{i \vec{k} \cdot \vec{x}}, \\
& \Pi_{1}(\vec{x})=\int \frac{d^{3} k}{(2 \pi)^{3}} \frac{-i}{\sqrt{2}}\left(a_{1}(\vec{k})-a_{1}^{\dagger}(-\vec{k})\right) e^{i \vec{k} \cdot \vec{x}},
\end{aligned}
$$

\footnotetext{
${ }^{5}$ This product inequality is satisfied if we use the euclidean norm, which, for a matrix $A$, is defined as the square-root of the largest eigenvalue of $A^{\dagger} A$.
} 


$$
\begin{aligned}
& \Phi_{2}(\vec{x})=\int \frac{d^{3} k}{(2 \pi)^{3}} \frac{-i}{\sqrt{2}}\left(a_{2}(-\vec{k})-a_{2}^{\dagger}(\vec{k})\right) e^{i \vec{k} \cdot \vec{x}} \\
& \Pi_{2}(\vec{x})=\int \frac{d^{3} k}{(2 \pi)^{3}} \frac{-1}{\sqrt{2}}\left(a_{2}(-\vec{k})+a_{2}^{\dagger}(\vec{k})\right) e^{i \vec{k} \cdot \vec{x}}
\end{aligned}
$$

we find that

$$
\hat{H}=\int_{\vec{p}}\left\{m\left(a_{1}^{\dagger}(\vec{p}) a_{1}(\vec{p})+a_{2}^{\dagger}(\vec{p}) a_{2}(\vec{p})\right)+i \sum_{j} \sin \left(p_{j}\right)\left(a_{1}(\vec{p}) \alpha^{j} a_{2}(\vec{p})-a_{2}^{\dagger}(\vec{p}) \alpha^{j} a_{1}^{\dagger}(\vec{p})\right)\right\},
$$

with $-\pi / 2<p_{j} \leq \pi / 2$. The $\alpha^{j}$ are anti-hermitian $8 \times 8$ Dirac matrices because there are $2^{3}$ spatial doublers. (The two doublers in in the time direction appear explicitly.) Now $\sum_{j} \sin \left(p_{j}\right) \alpha^{j}$ can be diagonalized, with eigenvalues

$$
\pm i s(p) \equiv \pm i \sqrt{\sum_{j} \sin ^{2}\left(p_{j}\right)}
$$

making $\hat{H}$ a sum of terms of the form

$$
h(\vec{p})=m\left(a_{1}^{\dagger}(\vec{p}) a_{1}(\vec{p})+a_{2}^{\dagger}(\vec{p}) a_{2}(\vec{p})\right) \pm s(p)\left(a_{1}(\vec{p}) a_{2}(\vec{p})-a_{2}^{\dagger}(\vec{p}) a_{1}^{\dagger}(\vec{p})\right) .
$$

For each $\vec{p}$, this can be diagonalized with a generalized Bogoliubov transformation [11]:

$$
\begin{aligned}
& b_{1}=\cos \theta a_{1}-\sin \theta a_{2}^{\dagger}, \\
& b_{2}=\cos \theta a_{2}-\sin \theta a_{1}^{\dagger}, \\
& \tilde{b}_{1}=\cos \theta a_{1}^{\dagger}+\sin \theta a_{2}, \\
& \tilde{b}_{2}=\cos \theta a_{2}^{\dagger}+\sin \theta a_{1},
\end{aligned}
$$

where we note that $\tilde{b}_{i}$ is not the hermitian conjugate of $b_{i}$. With $\theta=\frac{1}{2} \tan ^{-1}(s / m)$ this yields

$$
h=E\left(\tilde{b}_{1} b_{1}+\tilde{b}_{2} b_{2}\right), \quad E=\sqrt{m^{2}+s^{2}} .
$$

The operators $b_{i}$ and $\tilde{b}_{i}$ are annihilation and creation operators, and indeed, $\hat{H}$ has complete sets of left and right eigenstates. The form of the eigenvalues $E$ are not a surprise: the ghost determinant should cancel the valence determinant, so we expect the eigenvalues for the ghost hamiltonian to match those of the valence hamiltonian.

We can also calculate correlation functions in the theory with a trivial background. The twopoint functions in a theory with hamiltonian (3.3) are

$$
\begin{aligned}
\left\langle a_{i}(t) a_{j}^{\dagger}(0)\right\rangle & =\delta_{i j} \frac{E+m}{2 E} e^{-E t}, \\
\left\langle a_{i}^{\dagger}(t) a_{j}(0)\right\rangle & =-\delta_{i j} \frac{E-m}{2 E} e^{-E t}, \\
\left\langle a_{i}(t) a_{j}(0)\right\rangle & =-\left\langle a_{i}^{\dagger}(t) a_{j}^{\dagger}(0)\right\rangle=\delta_{i+j, 3} \frac{s}{2 E} e^{-E t} .
\end{aligned}
$$

While these correlation functions exhibit the expected exponential decay, they also clearly show a violation of unitarity. In a healthy field theory, by inserting a complete set of states between the operator at time $t$ and the one at time 0 , one would conclude that $\left\langle a_{i}^{\dagger}(t) a_{j}(0)\right\rangle$ has to be nonnegative. Here this is not the case, because the vacuum bra $\langle 0|$ defined by $\langle 0| \tilde{b}_{i}=0$ is not the conjugate of the vacuum ket $|0\rangle$ defined by $b_{i}|0\rangle=0$ since $\tilde{b}_{i}$ is not the hermitian conjugate of $b_{i}$. The results (3.8) also follow directly from a path integral for this hamiltonian, without first performing the Bogoliubov transformation. 


\section{Discussion}

In this talk, we took the first, and most important, step toward the construction of a transfer matrix for PQQCD. We considered PQQCD with staggered fermions, because it is straightforward to define the ghost sector in that case. The ghost quarks are then "staggered ghosts," i.e., staggered quarks with bosonic statistics, and we constructed the transfer matrix for staggered ghosts in an arbitrary gauge field background. The rest of the construction remains to be done, but we anticipate that adding the sea and valence parts, as well as the gauge part, to the transfer matrix will be straightforward.

We then proved that the ghost transfer matrix in an arbitrary gauge field background is bounded in the sense that the absolute value of its largest eigenvalue is bounded by one. At nonzero quark mass, correlation functions of ghost quarks are thus expected to decay exponentially, even if they do not satisfy the positivity properties of a healthy quantum field theory. We would expect this to carry over to PQQCD as long as that theory has a nonvanishing mass gap. In the case of PQQCD, the meaning of this would be that there is a unique eigenvalue $\lambda_{0}$ of the complete transfer matrix of the partially quenched theory with $\left|\lambda_{0}\right|$ maximal, while all other eigenvalues $\lambda$ satisfy $|\lambda|<\left|\lambda_{0}\right|$.

We then checked this in the free ghost theory, in the limit of vanishing temporal lattice spacing. Indeed, we find that there is a nonzero mass gap, set by the quark mass $m$, and all two-point functions decay exponentially. This supports the conjecture that the argument of Ref. [6] for the validity of ChPT can be carried over to the partially quenched case. Already in the free theory we find violations of unitarity in two-point functions, so it is clear that also PQQCD, and therefore PQChPT, will suffer from the same disease. However, PQChPT would still be the correct effective theory for PQQCD at low energy, because the underlying theory is local, and still satisfies cluster decomposition, despite its nonhermitian transfer matrix.

We thank Michael Ogilvie for helpful discussions.

\section{References}

[1] C. W. Bernard and M. F. L. Golterman, Phys. Rev. D 49, 486 (1994) [arXiv:hep-lat/9306005].

[2] S. R. Sharpe and N. Shoresh, Phys. Rev. D 62, 094503 (2000) [arXiv:hep-lat/0006017].

[3] O. Bär, G. Rupak and N. Shoresh, Phys. Rev. D 67, 114505 (2003) [arXiv:hep-lat/0210050].

[4] For a review of the applications of ChPT to lattice QCD, including PQChPT, see M. Golterman, arXiv:0912.4042 [hep-lat].

[5] S. Weinberg, Physica A 96, 327 (1979).

[6] H. Leutwyler, Annals Phys. 235, 165 (1994) [arXiv:hep-ph/9311274].

[7] S. R. Sharpe and N. Shoresh, Phys. Rev. D 64, 114510 (2001) [arXiv:hep-lat/0108003].

[8] C. Vafa and E. Witten, Nucl. Phys. B 234, 173 (1984); O. Bär, M. Golterman and Y. Shamir, in preparation.

[9] M. Golterman, S. R. Sharpe and R. L. Singleton Jr., Phys. Rev. D 71, 094503 (2005) [arXiv:hep-lat/0501015].

[10] J. Smit, unpublished lecture notes.

[11] M. S. Swanson, J. Math. Phys. 45, 585 (2004). 